\title{
Audience Gatekeeping in der Wirtschaftskommunikation: der Fall Greensill
}

\author{
Stefan Gürtler*
}

Soziale Medien ermöglichen es ibrem Publikum, Informationen zu liken, zu kommentieren und zu teilen. Nutzerinnen und Nutzer werden so selbst zum Informations-Gatekeeper, der Aufmerksamkeiten im Netzwerk auf bestimmte Themen und Ereignisse lenkt. Solche „Audience Gatekeeping “-Vorgänge wurden bisher in der Politik, kaum aber in der Wirtschaft untersucht. Gelegenheit dazu bietet die Insolvenz des Lieferkettenfinanzierers Greensill Capital im Frübling 2021. Anders als es politische Untersuchungen nabelegen, fübrte Audience Gatekeeping bei diesem Wirtschaftsereignis nicht zu einer alternativen Themendarstellung, die von der journalistischen Berichterstattung abwich, sondern stärkte die bestehende Informationshierarchie mit wenigen internationalen Leitmedien, die das Thema strukturierten. Gemeinsam mit den politischen Untersuchungen ist jedoch der Befund, dass Suchmaschinen und deren bierarchische Informationsauflistungen das Audience Gatekeeping beeinflussen und dass sich aus der Aufmerksamkeitslenkung qua Verlinkung eine extreme Ungleichverteilung zugunsten einer Handvoll Titel ergibt: Von insgesamt 943 verlinkten Medien erbielten drei Medientitel ein Viertel aller Links, während die Hälfte aller Quellen nur einmal verlinkt wurde. Einer proportionalen Power-Law-Verteilung, wie sie in verschiedenen Studien zur Medienaufmerksamkeit festgestellt wurde, folgt dieses Verteilmuster jedoch nur in abgeschwächter Form.

Schlüsselwörter: Greensill Capital, Twitter, Wirtschaftskommunikation, Kommunikationsereignis, Audience Gatekeeping, Gatewatching, Aufmerksamkeitsteuerung, Interaktions-Effekt, PageRank-Effekt, Domänen-Effekt, Rezyklierungs-Effekt, Negative Binomiale Regression, Power-Law-Verteilung

\section{Audience Gatekeeping in Business Communication: The Case of Greensill}

Social media empower their audience to like, comment, and share information. In this way, users become information gatekeepers who direct the attention of the network to certain topics and events. These "audience gatekeeping" processes have been studied quite extensively in the political realm, but hardly any studies refer to the business world. The insolvency of the financial services company Greensill Capital in spring 2021 provides an opportunity to apply this idea to the private sector. Contrary to what political research suggests, audience gatekeeping did not lead to an alternative presentation of issues but, rather, strengthened existing information hierarchies. Common to the political studies, however, is the finding that the direction of users' attention leads to the extremely unequal distribution of just a handful of titles: Of a total of 943 linked media titles, one quarter of the links connected to just three media titles while half were linked to only once. However, this distribution pattern follows a proportional power-law distribution, as has been found in various studies on media attention, only in a less pronounced form.

Keywords: Greensill Capital, Twitter, business communication, communication event, audience gatekeeping, gatewatching, attention control, interaction effect, PageRank effect, domain effect, recycling effect, negative binomial regression, power law distribution

* Prof. Dr. Stefan Gürtler, Fachhochschule Nordwestschweiz Hochschule für Wirtschaft, Riggenbachstrasse 16, 4600 Olten, Schweiz, stefan.guertler@fhnw.ch, ORCID https://orcid.org/ 0000-0002-9594-1078. 


\section{Einleitung}

Im Frühling 2021 gehörten die Schlagzeilen der Finanztitel dem „Aufstieg und Fall eines Fintech-Stars“ (Blume, Kröner, Volkey 2021): Der australisch-britische Lieferkettenfinanzier Greensill Capital und seine Tochtergesellschaften stellten Anfang März Antrag auf Insolvenz. Das Greensill-Geschäftsmodell bestand darin, gegen einen kleinen Nachlass Lieferantenrechnungen an Unternehmen rasch zu bezahlen. Um Liquidität zu generieren, wurden kurzfristige Forderungen in Wertpapiere verpackt und Anlegern zum Kauf angeboten. Am 1. März wurden Zeichnung und Rückgabe der Wertpapiere gesperrt, die Greensill gemeinsam mit dem Schweizer Bankhaus Credit Suisse vertrieb. Am 3. März schloss die deutsche Bafin wegen drohender Überschuldung die in Bremen ansässige Tochtergesellschaft Greensill Bank, die als Refinanzierer der Greensill-Gruppe sowie als Investor in Working Capital Solutions und Anbieter von Tages-, Festgeld- und Terminanlagen arbeitete. Das Unternehmen beantragte am 8. März 2021 Insolvenz. Die rasche, überraschende und unvorhersehbare Ereignisfolge, der hohe finanzielle Schaden und die Verstrickung prominenter Personen in die Insolvenz erzeugten ein anhaltendes mediales Interesse an den Vorgängen. Bedeutungssteigernd wurde die Greensill-Insolvenz als Sinnbild der „sich häufenden... Unfälle am Finanzmarkt“ (Ferber 2021: 19) und als unübersehbares Warnsignal für Systemrisiken (Wilson 2021) dargestellt, das sich einreihte in eine Kette ähnlicher symptomatischer Vorfälle wie Archegos oder Wirecard.

Die anhaltende Berichterstattung über die Implosion des Lieferkettenfinanzierers lässt sich als Kommunikationsereignis verstehen. Diese „ergeben sich, wenn Medien aus aktuellem Anlass über einen gewissen Zeitraum hinweg über ein Thema berichten“ (Schneider, Eisenegger 2020: 101) und damit ein Schlaglicht auf ein übergeordnetes (politisches oder wirtschaftliches) Thema werfen (Birkland 1997). Ereignisse sind im Gegensatz zu ihrem übergeordneten Thema zeitlich und räumlich begrenzt (Kepplinger 2001, Soroka 2002), treten plötzlich auf (Martin 2001) und können, da sie ein zentrales Problem verkörpern, wegen ihrer Unmittelbarkeit, ihrem Human-Interest-Gehalt, d. h. der menschlichen „story behind the story“ (Hughes 1980), und ihrer potenziellen Schädlichkeit nicht von der öffentlichen Agenda ferngehalten werden (Bytzek 2008). Eine weitere Ereignis-Eigenschaft ist die „Nichtobtrusivität“ (Zucker 1978), der Umstand, dass es vom Publikum in der Regel nicht direkt, sondern nur über die Medien erfahren werden kann (McCombs 2004).

Da Ereignisse Publikums-Aufmerksamkeit und -Wirkung fokussieren und maximieren (Reddy 1991), eröffnet in diesem Insolvenz-Fall die Lenkung der Aufmerksamkeit auf regulatorische Aspekte die Chance auf ein „Window of Reform“ (Bytzek 2008), das allerdings nur so lange offenbleibt, als neue Nachrichten zum Fall präsentiert werden können (ebd.). Da durch die Insolvenz auch beteiligte Banken zu Schaden kamen (Blume, Kröner, Volkey 2021), scheint die Berichterstattung zumindest in der Schweiz und zumindest bezüglich der Liquiditätsregeln für systemrelevante Banken ein „Window of Reform“ in Gang gebracht zu haben, wobei weniger eine Reform als vielmehr eine konsequentere Durchsetzung bestehender Regeln zur Debatte stand (Schöchli 2021).

Um das mediale Greensill-Ereignis genereller zu fassen: Was die Medien über derartige wirtschaftliche „Focusing Events“ (Birklund 1997) berichten, hat einen erheblichen Einfluss auf Wissen, Erwartungen, Meinungen und Handlungen von Wirtschaftsakteuren (Strycharz, Strauss, Trilling 2018; Tetlock 2014). „Wirksam“ werden allerdings nur jene Informationen, die in der täglichen Ereignisflut als berichtenswert eingestuft werden und zu den Akteuren gelangen (Da, Engelberg, Gao 2011; Pollock, Rindova 2003; Tetlock 2007). Damit rückt auf der Seite des Informationsangebots der Prozess der Informationsauswahl in den Fokus, der die Meinungsbildung über Ursachen und Ver- 
antwortlichkeiten im Ereignisfall formt und wirtschaftliche und politische Konsequenzen anstößt - oder diese verhindert.

Auf der Empfängerseite ist es die individuelle Kanal- und Themenwahl, das Medienrepertoire, welches darüber bestimmt, ob ein Kommunikationsereignis wahrgenommen und als wichtig erachtet wird (Schneider, Eisenegger 2020). Das Interesse an Wirtschaftsereignissen konzentriert sich auf ein zahlenmäßig stabiles Publikumssegment, das sich aus Qualitätsmedien informiert, bzw. ein wachsendes Segment mit einem digitalen und internationalen Medienrepertoire (Schwaiger, Schneider, Vogler 2020). Als Qualitätsmedien im Zusammenhang mit Wirtschaftsnachrichten gelten Zeitungen und Zeitschriften (Förster, Happ, Walstad 2019; Lindmayer, Dietz 2001; Riffe, Reader 2007). Soziale Medien gewinnen als Nachrichtenkanäle in der Wirtschaft an Popularität (Ranjan, Sood 2019), die Nachrichtenlage wird im Regelfall jedoch von der Berichterstattung der etablierten Wirtschaftsmedien und Wirtschaftsinformationsdienste geprägt (Strauss, Vliegenthart, Verhoeven 2017). Außergewöhnliche Kommunikationsereignisse können das Publikum jedoch dazu veranlassen, Informationen jenseits ihres Medienrepertoires in neuen Kanälen von neuen Quellen zu suchen (Hoskins, O’Loughlin 2011).

\section{Stand der Forschung}

Im Zuge der Digitalisierung des Nachrichtenwesens sind Selektionsprozesse auf Seiten des Informationsangebots eingehend untersucht worden. In der vordigitalen Zeit besaßen Druck- und elektronische Medien und ihre Medienhäuser ein Quasimonopol auf das Durchschleusen von Informationen an das Publikum, was ihnen eine einmalige gesellschaftliche Machtposition als „Informations-Gatekeeper“ verlieh: Wer Nachrichten verbreiten, seine Meinung äußern und seine Interessen signalisieren wollte, war auf Anerkennung durch die medialen Schleusenwärter angewiesen (Jungherr, Schroeder 2021). Dies galt in erster Linie für den Bereich der Politik, aber ebenso für alle anderen Gesellschaftsbereiche, in denen Akteure öffentliche Sichtbarkeit erlangen wollen.

Digitale Technologien „rekonfigurierten“ (ebd.) diesen Gatekeeping-Prozess, indem sie die Knappheit an Raum und Publikationskanälen sowie den unidirektionalen Informationsfluss aufhoben (Coddington, Holton 2014; Vos 2015). Neue Kanäle und Medienformen entzogen den „primären“ Gatekeepern ihre Vormachtstellung und eröffneten, zumindest theoretisch, jedem User gleiche Chancen auf Kommunikationsteilhabe (Ernste 2014). Praktisch schufen sie jedoch Platz für „sekundäre“ Gatekeeper, die „allen helfen wollen, die Welt zu verstehen, indem Menschen mit relevanten und autoritativen Nachrichten von einer Auswahl vertrauenswürdiger Partner" verbunden werden", wie es bei Google News heißt. ${ }^{1}$ Diese Auswahl erfolgt entweder link-basiert entsprechend der Verlinkungshäufigkeit auf digitale Beiträge, affinitäts-basiert entsprechend dem Informationsverhalten im Netzwerk oder publikums-basiert als Konsequenz von Nutzerinteraktionen rund um Information (Nielsen 2014), wobei im link- und affinitätsbasierten Gatekeeping typischerweise algorithmische Selektionsmechanismen zum Einsatz kommen (z. B. durch PageRank, RankDex-, TrustRank- oder EdgeRank-Algorithmen).

Sekundäre Gatekeeper umgehen in der Regel nicht die etablierten Gates, die von den primären Gatekeepern errichtet wurden, sondern selektieren die veröffentlichten Informationen nach eigenen (humanen oder algorithmischen) Relevanz- und Aktualitätskriterien. Eine zentrale Rolle spielt der Hyperlink, der als digitaler Querverweis das $\mathrm{Pu}-$ blikum per Mausklick zu externen Nachrichten bringt (Halavais 2008). Mit dem Hy-

1 https://support.google.com/news/publisher-center/thread/117443416/answers-to-some-com mon-questions-about-appearing-in-google-news?hl=en, Übersetzung SG [01.11.2021]. 
perlink hat sich ein Selektionsverfahren etabliert, bei dem die kollektive Verlinkung auf bestehende Informationen deren Zirkulation steigert (Goode 2009). Ein solches „Audience Gatekeeping“, das sich an bestehende Gates anschließt, lässt sich passenderweise mit Gatewatching umschreiben (Pałka-Suchojad 2021; Welbers 2018). Seine Wirkung besteht darin, dass das Publikum durch die Leseempfehlungen seiner Peers in der Informationswahl beeinflusst wird (Dimitrova, Connolly-Ahern, Williams, Kaid, Reid 2003).

Audience Gatekeeping bzw. Gatewatching erweitert das Gatekeeping-Konzept in der Summe erstens durch eine neue Gattung von Gatekeepern, die sich aus dem Publikum selbst rekrutieren, was zweitens die Trennung zwischen Informationslieferanten und -empfängern verwässert. Drittens zielt Audience Gatekeeping weniger auf die redaktionelle Aufbereitung und Platzierung von Inhalten, sondern auf deren Gewichtung durch Klicks, Likes, Kommentierung und Verlinkung. Viertens wird die Aufmerksamkeit nicht innerhalb eines Mediums, sondern auf beliebige Medien umgelenkt (Leavitt, Robinson 2017). Die sozialen Verlagerungen zwischen Sender und Empfänger führen fünftens zu inhaltlichen Anpassungen, da die „Weiterleitungswahrscheinlichkeit“ durch das Publikum zu einem dominanten Inhaltskriterium wird (Keyling 2017).

Über die medialen und gesellschaftlichen Konsequenzen dieses Strukturwandels in der Informationsselektion gehen die Meinungen auseinander. Auf der einen Seite wird auf die Demokratisierung-, Dezentralisierungs-, Disintermediations- und Partizipationsgewinne im Mediensystem verwiesen (Benkler 2006; Xu, Feng 2014). Verschiedene Studien belegen eine Machtverschiebung von den etablierten Medien zu den „networked publics“ (Meraz, Papacharissi 2016), die in der Darstellung politischer Ereignisse andere Akzente setzen als die etablierten Medien (Demirhan 2014; Fletcher, Park 2017; Kwon, Oh, Agrawal, Rao 2012; Meraz, Papacharissi 2013; Neuman, Guggenheim, Jang, Bae 2014; Strauss 2018).

Kritische Stimmen zur „Rekonfiguration“, die seit den US-Präsidentenwahlen 2016 überhandgenommen haben (Freelon, Wells 2020), halten entgegen, dass digitale Medien nur den Zugang zur öffentlichen Arena erleichtern, nicht aber gesellschaftliche Anerkennung verleihen. Gehört werden auch weiterhin nur wenige (Neuman, Bimber, Hindman 2011), wie bislang braucht es die etablierten Medien (Karpf 2010) oder Online-Hubs (Hindman 2008), um Themen und Anliegen aufzunehmen und in der Öffentlichkeit bekannt zu machen. Die Kontrolle der Informationsflüsse bleibt, in modifizierter Form, in der Hand weniger Gatekeeper. Eine zweite Argumentationslinie bestreitet zwar nicht den Strukturwandel im Gatekeeping, moniert aber, das neue Gatekeeper vor allem auf den Plan getreten sind, um ungehindert Falschnachrichten und extreme Meinungen in die Öffentlichkeit einschleusen zu können (Marantz 2019; Pomerantsev 2019; Singer, Brooking 2018).

\section{Forschungslücke}

In den erwähnten Studien wird Gatekeeping explizit oder implizit als politischer Prozess mit dem Ziel der Informationskontrolle verstanden (Barzilai-Nahon 2008, 2009) und ausschließlich auf Ereignisse der politischen Kommunikation angewendet. Eine solche Einschränkung ist nicht zwingend: Chakraborty, Ghosh, Ganguly, Gummadi (2019) zeigen anhand von Leseempfehlungen für Artikel der New York Times, dass Audience Gatewatching auf Social-Media-Plattformen auch nichtpolitische Themenfelder betrifft: Auf Twitter sind es Artikel aus den Sparten „Business“ und „World“, auf Facebook dagegen „Opinions“ und „Local Stories“. Es ist deshalb naheliegend, Gates und Gate- 
keeper hinter sämtlichen gesellschaftsrelevanten Informationsflüssen zu vermuten, so auch für die Wirtschaftskommunikation.

Ein Medium par excellence zum Studium von Audience-Gatekeeping-Prozessen ist Twitter. Dieser Dienst ermöglicht das Verfassen und Verbreiten von Kurzbotschaften, die mit einem Hyperlink als Verweis auf externe Informationen versehen werden können. Knappe Form und externe Informationsverlinkung haben Twitter zu einem bevorzugten Instrument der Informationsbrokerage in Politik, Wirtschaft oder Sport gemacht (Cha, Haddadi, Benevenuto, Gummadi 2010). Unter anderem weisen Studien eine Granger-Kausalität zwischen der Stimmung auf Twitter und der Stimmung an den Aktienmärkten nach (Bharathi, Geetha 2017; Bharathi, Geetha, Sathiynarayanan 2017; Zhao, He, Yuan, Huang 2016; Sun, Wang, Zhang, Cao, Liu, Wang 2017). Der Dienst ist ein bevorzugtes Instrument des Informationsaustauschs zwischen Börsenhändlern (Caliñgo, Sison, Tanguilig 2016) oder - wie die Twitter-Tätigkeit von Elon Musk exemplarisch zeigt-der manipulativen Marktbeeinflussung (Case 1: 18-cv-01669-UNA 2018; Grant, Eisenhofer 2019; Honegger 2021; Mulvaney, Baldo, Swerdlow, Cavaliere 2018).

Die vorliegende Studie schließt eine Forschungslücke, da erstmalig Audience Gatekeeping-Vorgänge rund um Wirtschaftsereignisse Gegenstand der Untersuchung sind.

\section{Forschungsfragen und methodischer Steckbrief}

\subsection{Forschungsfragen und Hypothesen}

In seiner Anlage folgt die vorliegende Untersuchung der Studie von Kwon, Oh, Agrawal, Rao (2012), welche Audience Gatekeeping im Fall des Gaza-Konflikts 2009, einem politischen Kommunikationsereignis also, untersuchte. Die Autoren kamen darin erstens zum Schluss, dass die Informationsauswahl des Twitter-Publikums zwar ein breites Spektrum an Onlinequellen umfasste, aber eine kleine Anzahl prominenter Websites überrepräsentiert war, und zwar in einem proportionalen Power-Law-Verhältnis. Zweitens stellten sie fest, dass die Informationsauswahl nicht zufällig erfolgte, sondern von der Anzahl der Backlinks und der Art der Informationskanäle beeinflusst wurde. Drittens konnten sie bestätigen, dass die etablierten Medien keine dominante Stellung als Informationsquellen im Konflikt hatten, dass politische Informationselektion tatsächlich von Demokratisierungs- und Dezentralisierungstendenzen gezeichnet war.

Um in ähnlicher Weise die Breite der Informationsauswahl und die Aufmerksamkeitsverteilung auf mediale Quellen im Fall eines Wirtschaftsereignisses zu prüfen, lautet

\section{Forschungsfrage 1: Werden für das Audience Gatekeeping bei Wirtschaftsereignissen etablierte Wirtschaftsmedien bevorzugt?}

Es ist anzunehmen, dass Berichte über wirtschaftliche Ereignisse die Domäne der etablierten Wirtschaftsmedien sind, schon aufgrund ihrer Vernetzungs- und Kompetenzvorteile bei der Recherche und Einordnung dieser Informationen, und dass sie die Vorteile dieser Informations-Asymmetrie zu ihren Gunsten nutzen (Stieglitz 2002). Unklarheiten über Ursachen, Folgen und Verantwortlichkeiten machen die Greensill-Insolvenz zu einem investigativen Thema, was die redaktionellen Kapazitäten etablierter Wirtschaftsmedien bevorteilen dürfte. Es erscheint daher wenig wahrscheinlich, dass andere Medien und Akteure außerhalb dieser Domäne wesentliche Neuigkeiten zum Fall beisteuern und die Informationshierarchie auf den Kopf stellen können.

Das Publikum dürfte deshalb bevorzugt Informationen aus der Domäne der Wirtschaftsmedien beziehen, in Sinne von 
Hypothese 1.1: Der Medientyp beeinflusst die Zabl der Hyperlinks auf ein Medium (Domänen-Effekt).

Kwon et al. (2012) argumentieren in ihrer Studie, dass Twitter-Accounts einen Reputationsgewinn erzielen können, wenn sie auf relevante, bisher unbekannte Informationsquellen verlinken können. Die Suchkosten nach Informationen zu einem Ereignis werden dann unter einer großen Anzahl von Accounts amortisiert, indem sowohl leicht auffindbare als auch obskure Informationsquellen verlinkt werden. Es ist anzunehmen, dass bei einem Kommunikationsereignis dieses Ausmaßes in alternativen Kanälen und von alternativen Quellen nach Informationen zu Greensill gesucht wird (Hoskins, O’Loughlin 2011). Dabei werden Verlinkungen auf Informationsquellen dominieren, die das Publikum bei seiner eigenen Recherche an prominenter Stelle in Suchmachinenergebnissen vorfindet (Israel, Samson 2018). Das Ranking der Informationen wird wesentlich mitbestimmt von den Backlinks, die eine Quelle von Dritten erhält (Banerjee, Choudhary 2017; zum Begriff und zur Messung siehe weiter unten). Akzeptiert man Backlinking als Gradmesser für mediale Popularität (ebd.) und hohes Ranking als Gradmesser für (mediale) Relevanz (Poonkuzhali, Kishore Kumar, Sudhakar, Uma, Sarukesi 2012), lautet

Hypothese 1.2: Die Verlinkungs-Häufigkeit eines Mediums bzw. Medieninhalts steht in Zusammenhang mit dessen (Suchmaschinen-)Relevanz (PageRank-Effekt).

Die Untersuchung von Kwon et al. (2012) legt nahe, dass die absolute Rangierung in den Suchergebnissen (PageRank-Effekt) von der Relevanz der Inhalte und der Themenkompetenz der Informationsanbieter relativiert wird. Tiefer rangierte Suchtreffer themenkompetenter Medientypen (im Fall von Wirtschaftsereignissen beispielsweise von Wirtschaftsmedien) würden gegenüber höher rangierten Suchtreffern von weniger themenkompetent eingeschätzten Medientypen bevorzugt. Es resultiert deshalb eine Interaktion zwischen PageRank- und Domänen-Effekt, im Sinne von

\section{Hypothese 1.3: Die Verlinkungshäufigkeit unterliegt der Wechselwirkung von Medien- typ und Popularität seiner Inhalte (Interaktions-Effekt).}

Gemäß Strauss et al. (2017) haben sich die bekannten Wirtschaftsmedien erfolgreich im digitalen Raum positioniert. Es ist deshalb anzunehmen, dass im wirtschaftlichen Ereignisfall wenige Akteure und Medien die Themenagenda bestimmen und die Aufmerksamkeit des Publikums für sich maximieren können. Dies gibt Anlass zur

Forschungsfrage 2: Fübrt Audience Gatekeeping zu einer Inbaltskonzentration zugunsten weniger, einflussreicher Schlüsselmedien?

Teil der oben erwähnten erfolgreichen Positionierung sind Anpassungen, die etablierte Medien an ihrer Informationsselektion und -präsentation vorgenommen haben, um in digitalen Kanälen Aufmerksamkeit und Relevanz zu erzeugen (Mitchelstein, Boczkowski 2009). In diesem Rahmen sind sie zu „Informationsintermediären“ ihrer selbst geworden, die dem Publikum in Rubriken wie „Meistgelesen“ oder „Meistgeteilt“ ein sekundäres Gatewatching der eigenen Inhalte anbieten (Smyrnaios 2012). Es ist deshalb naheliegend, dass Medien in den Sozialen Medien ihren erfolgreichen Content rezyklieren, um ihre Informationsbeschaffungs- und Verteilungskosten zu senken und ihre Sichtbarkeit und Bekanntheit als Informationsquellen in den Sozialen Netzwerken zu erhöhen. Deshalb lautet

Hypothese 2.1: Medien nutzen Verlinkungen auf frübere Tweets zur Rezyklierung und Beachtungssteigerung ibres eigenen Contents (Rezyklierungs-Effekt). 
Die Kumulation aller genannten Effekte - Domänen-, PageRank-, Interaktions- und Rezyklierungs-Effekt - dürfte zu einer extremen Ungleichverteilung der Aufmerksamkeitslenkung führen, wobei wenig Aufmerksamkeit auf viele Titel und viel Aufmerksamkeit auf wenige Titel gelenkt wird. Da im digitalen Raum die verfügbare Aufmerksamkeit ein knappes Gut bleibt (Wiedel 2015), bedeutet jeder zusätzliche Link für einen etablierten Titel, dass ein anderer Titel, der ebenfalls über das Ereignis berichtet, aus dem Gesichtskreis des Publikums verschwindet und damit zur „unsichtbaren“ Information wird. Deshalb lautet

Hypothese 2.2: Die Aufmerksamkeitsverteilung im wirtschaftlichen Ereignisfall folgt dem Potenzgesetz, indem in proportionalem Verbältnis sebr wenige Medien sebr häufig verlinkt werden und sebr viele Medien sebr selten.

\subsection{Untersuchungssteckbrief}

Als Untersuchungszeitraum wurde der 11.-19. März 2021 gewählt, eine Woche nach der Insolvenz, als eine breite Debatte zu Ursachen, Folgen und Verantwortlichkeiten eingesetzt hatte.

Die Twitterdebatte wurde mit dem Python-Tweepy-Modul über die Search-API (Query: „greensill“) heruntergeladen. Tweets, die Verweise auf externe Medien enthielten (Variable „entities\{urls“) wurden extrahiert und die gekürzten URLs über eine requests-Anfrage expandiert. Die Accounts wurden auf der Basis ihres Namens und ihrer Selbstbeschreibung (Variable „user\{description“) in private und nicht-private Accounts klassifiziert. Dazu wurden Accountnamen und -beschreibungen mit einem Sequence Matching-Verfahren separiert. ${ }^{2}$

Die Zuweisung der Hyperlinks auf verschiedene Medienkanäle (vgl. Tabelle 1) erfolgte in Anlehnung an Kwon et al. (2012). Da ein wirtschaftsaffines Publikum sich breit informiert, einerseits aus etablierten Qualitätsmedien und andererseits aus digitalen Informationsangeboten (Schwaiger et al. 2020), ist ein umfangreiches Medienrepertoire zu erwarten, das beim Gatewatching herangezogen wird. Um die fortschreitende „Plattformisierung der Mediennutzung “ (fög 2019) zu berücksichtigen, also das Aufkommen von Medien mit algorithmischem Gatewatching aus verschiedenen Quellen, wurden „gebündelte“ Informationsangebote etablierter Medien und „entbündelte“, plattformisierte Informationsangebote von News-Aggregatoren und Sozialen Medien, kurz: neuer Medien, unterschieden. Diese Zuteilung erfolgte mit einem String-Matching-Verfahren, da die meisten Medien im Untersuchungsset in der Description auf ihre publizistischen

2 Verwendet wurde ein difflib SequenceMatcher, der Screen-Name und Description eines Accounts miteinander verglich. Bei Inhaltsübereinstimmung von $\geq 0.5$ mindestens eines Wortes wurde auf einen nicht-privaten, bei Verwendung von geläufigen männlichen und weiblichen Vornamen in der Variable „user\{screen_name“ auf einen privaten Account geschlossen. Ankerbeispiel für einen institutionellen Account: „user\{screen_name“: „finews_ch“; „user\{description“: „finews.ch provides regular news coverage from the Swiss finance sector, as well as banking news from Singapore and Hong Kong with https://t.co/DeOgcx9ACv.“ - Account-Name wird in der Description wiederholt. Die so codierten Daten dienten als Training-Set für ein maschinelles Text-Klassifizierungsverfahren, das die Descriptions der noch unklassifizierten Accounts mit jenen der klassifizierten Accounts verglich (Datentransformation mit Count Vectorizing, anschließende logistische Regression mit saga-Solver). Die Zuweisungsgenauigkeit (accuracy) lag bei 94.09 Prozent. 
Aktivitäten hinwiesen. ${ }^{3}$ Nicht per Matching zuzuordnende Domains wurden manuell nachcodiert. Bei den Sozialen Medien wurden nicht die Plattformen als Gatekeeper behandelt, sondern die einzelnen User (Jürgens, Jungherr, Schoen 2011; Mehrotra 2017). Dies ergibt die in Tabelle 1 dargestellte Medientypologie.

\section{Tabelle 1: Medientypen}

\begin{tabular}{llll}
\hline Nr. & Medientyp & Beschreibung & Beispiel \\
\hline 1 & $\begin{array}{l}\text { Etablierte } \\
\text { Wirtschaftsmedien }\end{array}$ & $\begin{array}{l}\text { Nachrichtendienste, Druck-, elektronische Medien } \\
\text { mit redaktionellen Beiträgen zum } \\
\text { Themenschwerpunkt Wirtschaft. }\end{array}$ & $\begin{array}{l}\text { ft.com } \\
\text { bloomberg.com }\end{array}$ \\
\hline 2 & $\begin{array}{l}\text { Neue } \\
\text { Wirtschaftsmedien }\end{array}$ & $\begin{array}{l}\text { Internet-Angebote mit aggregierten Beiträgen zu } \\
\text { Wirtschaftsthemen. }\end{array}$ & $\begin{array}{l}\text { inkl.com } \\
\text { thehustle.com }\end{array}$ \\
\hline 3 & $\begin{array}{l}\text { Etablierte } \\
\text { Publikumsmedien }\end{array}$ & $\begin{array}{l}\text { Nachrichtendienste, Druck-, elektronische Medien } \\
\text { mit redaktionellen Beiträgen zu allen } \\
\text { Gesellschaftsthemen. }\end{array}$ & $\begin{array}{l}\text { tagesanzeiger.ch } \\
\text { tagesschau.de }\end{array}$ \\
\hline 4 & $\begin{array}{l}\text { Neue } \\
\text { Publikumsmedien }\end{array}$ & $\begin{array}{l}\text { Internet-Angebote mit aggregierten Beiträgen zu } \\
\text { allen Gesellschaftsthemen. }\end{array}$ & $\begin{array}{l}\text { thetop10news.com } \\
\text { nuzzel.com }\end{array}$ \\
\hline 5 & $\begin{array}{l}\text { Etablierte und neue } \\
\text { Fachmedien }\end{array}$ & $\begin{array}{l}\text { Medien und News-Aggregatoren, mit Redaktion } \\
\text { oder Aggregation zu Themenschwerpunkten } \\
\text { außerhalb der Wirtschaft. }\end{array}$ & $\begin{array}{l}\text { autocarnews.com } \\
\text { actuarialnews.com }\end{array}$ \\
\hline 6 & $\begin{array}{l}\text { Persönliche } \\
\text { Reports }\end{array}$ & $\begin{array}{l}\text { Persönliche News-Kurationen mit eigener } \\
\text { (Sub-)Domain zu allen Themen (u. a. Wirtschaft). }\end{array}$ & $\begin{array}{l}\text { da-via.com } \\
\text { wolfstreet.com }\end{array}$ \\
\hline 7 & $\begin{array}{l}\text { Institutionelle } \\
\text { Webseiten }\end{array}$ & $\begin{array}{l}\text { Informationen von Firmen, politischen Institutionen, } \\
\text { Interessengruppen ohne Kerngeschäft in } \\
\text { Informationsvermittlung. }\end{array}$ & devdiscourse.com \\
\hline
\end{tabular}

Die Popularität der referenzierten Medientitel (nicht der einzelnen Inhalte) wurde mittels Backlink-Tracking gemessen. Dieses ermittelt die Zahl der Seiten, die auf die referenzierten Domains (und Subdomains) verweisen. Backlinks erzeugen Webtraffic und erhöhen die Sichtbarkeit in Suchresultaten, wobei die Zahl der Backlinks und die Qualität der bezugnehmenden Second-Level-Domains (idealerweise .org oder .gov) als Faktoren in das Pageranking einfließen (Jain, Dave 2013; Ziakis, Vlachopoulou, Kyrkoudis, Karagkiozidou 2019). Die Backlink-Statistik wurde mit dem kommerziellen Dienst von www.ahrefs.com erstellt, da ein eigenes Link-Crawling (z. B. mit Python Scrapy) für die in dieser Studie erforderlichen 84 Milliarden Backlinks einen sehr hohen Zeit- und Rechenaufwand erfordert hätte. Ahrefs wurde in verschiedenen Untersuchungen zur Sichtbarkeit, Relevanz und Popularität von Webseiten genutzt (z. B. Patel, Trivedi, Bhatt, Shanti 2021; Seiawan, Harahap, Syamsuar, Kunang 2020; Suksida, Santiworarak 2017; Wahyuningrum, Pandiya 2017).

3 Ankerbeispiel: Webdomain = businesslive.co.za; user (description $=$ „Business Day is South Africa's best daily business newspaper, part of the BusinessLIVE network. We're also on Facebook and LinkedIn." 


\section{Ergebnisse}

\subsection{Frequenzen}

Im Untersuchungszeitraum wurden 11098 Tweets mit Stichwort „greensill“ erfasst, 5734 Tweets (51.7 \%) beinhalteten 6466 Links auf externe Informationsquellen. 5 Links ließen sich nicht expandieren, 12 führten nicht zu Informationen zu Greensill Capital, so dass 6449 Links auf 943 verschiedene Second-Level-Domains bzw. Quellen zur weiteren Analyse verblieben.

Von den 6449 Links verwiesen 64.5 Prozent auf etablierte Wirtschafts- und Publikumsmedien (Medientypen 1 und 3, vgl. Tabelle 2). Neue Wirtschafts- und Publikumsmedien, d. h. News-Aggregatoren ohne redaktionelle Eigenleistung (Medientypen 2 und 4), erhielten 11.3 Prozent der Links. 16.6 Prozent der Beiträge verlinkten auf andere Social-Media-Beiträge (Text-, Audio- und Videobeiträge auf Twitter selbst, Instagram, Soundcloud, Youtube usw.). Zahlenmäßig von geringerer Bedeutung waren Verlinkungen auf Fachmedien (2.4\%) und organisationale Webseiten von Firmen, Verbänden, Parteien und Interessengruppen (5.2\%).

Die Verlinkungen stammten zu 68.5 Prozent (4420) von privaten und zu 31.5 Prozent (2029) von institutionellen Accounts. Medienpräferenzen, d. h. Verlinkungsunterschiede, wurden mittels Chi-Quadrat-Kontingenzanalyse untersucht. Das Verfahren sucht nach signifikanten Unterschieden zwischen Erwartungs- und Beobachtungshäufigkeiten und ermittelt daraus einen etwaigen Zusammenhang zwischen den untersuchten $\mathrm{Va}$ riablen sowie mit Phi oder Cramer's V ein Maß für die Effektstärke (Backhaus, Erichson, Plinke, Weiber 2016). Zwischen Account-Typ und den Verlinkungen auf Medienkanäle besteht ein signifikanter Zusammenhang $\left(\chi^{2}=555.535, \mathrm{p}<0.001\right)$, jedoch mit (Berücksichtigung der Fallzahlen; vgl. Nelson, Wooditch, Dario 2014) geringer bis mittlerer Effektstärke $(\phi=0.293, p<0.001)$. Private Accounts liegen bei der Verlinkung auf SocialMedia-Beiträge anderer User über den Erwartungswerten, institutionelle Accounts bei der Verlinkung auf News-Aggregatoren (neue Wirtschafts- und Publikumsangebote) und organisationale Webseiten.

Tabelle 2: Hyperlinkverteilung auf Medien-und Accountkategorien; Medientypologie gemäß Tabelle 1

\begin{tabular}{|c|c|c|c|c|c|c|}
\hline \multirow{2}{*}{$\begin{array}{l}\text { Medientyp } \\
\text { Etablierte Wirtschaftsmedien }\end{array}$} & \multicolumn{2}{|c|}{$\begin{array}{l}\text { Institutionelle } \\
\text { Hyperlinks }\end{array}$} & \multicolumn{2}{|c|}{$\begin{array}{c}\text { Private } \\
\text { Hyperlinks }\end{array}$} & \multicolumn{2}{|c|}{$\begin{array}{c}\text { Hyperlinks } \\
\text { Total }\end{array}$} \\
\hline & 877 & $43.2 \%$ & 2054 & $46.5 \%$ & 2931 & $45.5 \%$ \\
\hline Neue Wirtschaftsmedien & 273 & $13.5 \%$ & 220 & $5.0 \%$ & 493 & $7.6 \%$ \\
\hline Etablierte Publikumsmedien & 414 & $20.4 \%$ & 810 & $18.3 \%$ & 1224 & $19.0 \%$ \\
\hline Neue Publikumsmedien & 132 & $6.5 \%$ & 104 & $2.4 \%$ & 236 & $3.7 \%$ \\
\hline Fachmedien & 69 & $3.4 \%$ & 89 & $2.0 \%$ & 158 & $2.4 \%$ \\
\hline Social-Media-Beiträge & 87 & $4.3 \%$ & 985 & $22.3 \%$ & 1072 & $16.6 \%$ \\
\hline Organisations-Medien & 177 & $8.7 \%$ & 158 & $3.6 \%$ & 335 & $5.2 \%$ \\
\hline Total & 2029 & $\begin{array}{l}100.0 \% \\
(31.5 \%)\end{array}$ & 4420 & $\begin{array}{l}100.0 \% \\
(68.5 \%)\end{array}$ & 6449 & $\begin{array}{c}100.0 \% \\
(100.0 \%)\end{array}$ \\
\hline
\end{tabular}




\subsection{Modell-Test}

Als ereignisbasiertes Zählverfahren für eine fixe Zeitperiode (Hyperlinks zu GreensillBerichten innerhalb einer Woche) kam eine negative Binomialregression ohne OffsetVariable zum Einsatz (Python-Modul Statsmodels). Diese ist eine Verallgemeinerung der Poisson-Regression ohne deren Äquidispersions-Annahme (Varianz entspricht Mittelwert; Coxe, West, Aiken 2009). Damit eignet sich die Binomialregression auch für Verteilungen mit Über- und Unterdispersionen, d. h. mit Varianz größer bzw. kleiner als Mittelwert (Hilbe 2014). Payne, Gebregziabher, Hardin, Ramakrishnan, Egede (2017) schlagen als Schwellenwert für eine solche Überdispersion eine Verhältniszahl aus der Abweichung zu ihren Freiheitsgraden und/oder des Pearson-Chi-Quadrats zu den Freiheitsgraden von > 1.2 vor. Der Anpassungsgüte-Test weist bei der Abweichung ein Wert/df-Verhältnis von 1025.760 / $929=1.104$ bzw. ein Pearson-Chi-Quadrat / df von 3884.883 / 992 = 4.182 aus. Es kann also eine Überdispersion angenommen werden, und die Anwendung einer negativen Binomialregression statt einer Poisson-Regression scheint angezeigt.

Ein allgemeiner Omnibus-Tests mit einem Likelihood-Ratio $\chi^{2}=889.881, p<0.001$ zeigt, dass ein Modell mit den beiden Prädiktoren „Medientyp“ und „Medienpopularität" für die nachfolgend durchgeführte Überprüfung des PageRank-Effekts signifikant besser abschneidet als ein Modell ohne Prädiktoren (Osborne 2017). Mit anderen Worten: Medientypologie und Medienpopularität sind geeignete Variablen, um Audience Gatekeeping-Prozesse zu erklären.

\subsection{Domänen-Effekt: Einfluss von Medientyp auf Verlinkungen}

Forschungsfrage 1 interessiert sich für die Muster der Medienauswahl beim Audience Gatekeeping, Hypothese 1.1 postuliert in diesem Zusammenhang, dass die Nutzerbeteiligung am Gatekeeping-Prozess nicht zu einer breiteren Aufmerksamkeitsverteilung führt, sondern im Gegenteil die etablierten Medientypen bevorzugt -im wirtschaftlichen Ereignisfall Berichte aus der Domäne der Wirtschaftsmedien.

Die Ergebnisse bestätigten den Medientyp (Wald $\chi^{2}=60.508, p<0.001$ ) und die Backlinks (Wald $\chi^{2}=4.455, p=0.035$ ) als signifikante Faktoren im Audience Gatekeeping (vgl. Tabelle 3). Da es sich beim Medientyp um ein kategoriales Merkmal handelt, war für die Ermittlung der Unterschiede zwischen den Medientypen ein Referenzniveau erforderlich. Als solches wurden die etablierten Wirtschaftsmedien gesetzt, basierend auf den anzunehmenden Berichterstattungsvorteilen im wirtschaftlichen Ereignisfall. Die Verlinkung tendierte zu Berichten der etablierten Wirtschaftsmedien, für die mehr Aufmerksamkeit geschaffen wurde als für die anderen Medientypen.

Aus Tabelle 3 geht hervor, dass - anders als in den bisherigen politischen Studien die etablierten Wirtschaftsmedien der wichtigste Kanal waren, auf welchen die Aufmerksamkeit anderer User gelenkt werden sollte. Unter Zuhilfenahme dieses Referenzniveaus lässt sich aus den Parameterschätzungen in Tabelle 3 das Ausmaß des „Aufmerksamkeitsnachteils" ermitteln, den die anderen Medientypen bei der Ereignisdarstellung gegenüber den Wirtschaftsmedien zu gewärtigen hatten. Verglichen mit den etablierten Wirtschaftsmedien (Inzidenzrate $\operatorname{Exp}(B)=1$ ), sank unter gleichen Bedingungen die Verlinkungswahrscheinlichkeit bei allen anderen Medientypen. Informationen von organisationalen Akteuren weisen beispielsweise eine Inzidenzrate (d. h. Verlinkungswahrscheinlichkeit) von 0.283 (verglichen mit 1.000 bei den etablierten Wirtschaftsmedien) aus. Anders ausgedrückt sank ihre Hyperlinking-Wahrscheinlichkeit um 72 Prozent $(\operatorname{Exp}(B) 0.283-\operatorname{Exp}(B)$ Referenzwert $1.000=-0.717)$. 
Tabelle 3: Parameterschätzung mit Bezugsebene etablierte Wirtschaftsmedien

\begin{tabular}{|c|c|c|c|c|c|c|c|c|c|}
\hline \multirow[b]{2}{*}{ Parameter } & \multirow[b]{2}{*}{$\beta$} & \multirow[b]{2}{*}{ S.E. } & \multicolumn{2}{|c|}{$\begin{array}{c}95 \% \text { Wald- } \\
\text { Konf.intervall }\end{array}$} & \multicolumn{2}{|c|}{ Hypothesentest $_{*}$} & \multirow[b]{2}{*}{$\operatorname{Exp}(B)$} & \multicolumn{2}{|c|}{$\begin{array}{l}95 \% \text { Wald- } \\
\text { KI für } \operatorname{Exp}(B)\end{array}$} \\
\hline & & & Unterer & Oberer & Wald $\chi^{2}$ & Sig. & & Unterer & Oberer \\
\hline Organisations-Medien & -1.263 & 0.1785 & -1.613 & -0.913 & 50.091 & 0.000 & 0.283 & 0.199 & 0.401 \\
\hline Social-Media-Beiträge & -0.889 & 0.1589 & -1.200 & -0.577 & 31.296 & 0.000 & 0.411 & 0.301 & 0.561 \\
\hline Fachmedien & -0.954 & 0.2722 & -1.488 & -0.420 & 12.281 & 0.000 & 0.385 & 0.226 & 0.657 \\
\hline Neue Publikumsmedien & -0.902 & 0.2097 & -1.313 & -0.491 & 18.499 & 0.000 & 0.406 & 0.269 & 0.612 \\
\hline Etablierte Publikumsmedien & -1.098 & 0.1942 & -1.479 & -0.718 & 31.992 & 0.000 & 0.333 & 0.228 & 0.488 \\
\hline Neue Wirtschaftsmedien & -0.566 & 0.1860 & -0.931 & -0.202 & 9.266 & 0.002 & 0.568 & 0.394 & 0.817 \\
\hline $\begin{array}{l}\text { Etablierte } \\
\text { Wirtschaftsmedien }\end{array}$ & $0 * *$ & & & & & & 1 & & \\
\hline Backlinks (10000) &, 001 &, 0001 &, 001 &, 001 & 85.161 & 0.000 & 1,001 & 1,001 & 1,001 \\
\hline $\begin{array}{l}\text { Organisations-Medien } \\
\text { "Backlinks }\end{array}$ &,- 001 &, 0002 &,- 002 &,- 001 & 27.703 & 0.000 & ,999 & ,998 & ,999 \\
\hline $\begin{array}{l}\text { Social-Media-Beiträge } \\
* \text { Backlinks }\end{array}$ &,- 001 & ,0004 &,- 002 &,- 001 & 13.110 & 0.000 & ,999 & ,998 & ,999 \\
\hline $\begin{array}{l}\text { Fachmedien } \\
* \text { Backlinks }\end{array}$ & ,002 & ,0022 &,- 002 &, 006 & 0.819 & 0.366 & 1,002 & ,998 & 1,006 \\
\hline $\begin{array}{l}\text { Neue Publikumsmedien } \\
\text { *Backlinks }\end{array}$ &,- 001 & ,0002 &,- 002 &,- 001 & 29.490 & 0.000 & ,999 & ,998 & ,999 \\
\hline $\begin{array}{l}\text { Etablierte Publikumsmedien } \\
\because \text { Backlinks }\end{array}$ &, 000 &, 0002 &,- 001 & $2,417 \mathrm{E}-5$ & 3.258 & 0.071 & 1,000 & ,999 & 1,000 \\
\hline $\begin{array}{l}\text { Neue Wirtschaftsmedien } \\
\text { "Backlinks }\end{array}$ &,- 001 &, 0002 &,- 001 &,- 001 & 37.565 & 0.000 & ,999 & .999 & .999 \\
\hline $\begin{array}{l}\text { Etablierte } \\
\text { Wirtschaftsmedien } \\
* \text { Backlinks }\end{array}$ & $0 * * * \%$ & & & & & & 1 & & \\
\hline
\end{tabular}

$* \mathrm{df}=1 ; *$ Etablierte Wirtschaftsmedien als Bezugsebene; *** Etablierte Wirtschaftsmedien $\mathrm{x}$ Backlinks als Bezugsebene

Die Hypothese 1.1 kann somit bestätigt werden.

\subsection{PageRank-und Interaktions-Effekt: Einfluss von Medienpopularität auf Verlinkungen}

Hypothese 1.2 postuliert, dass beim Audience Gatekeeping tendenziell auf populäre, sprich: leicht auffindbare, in Suchmaschinen hoch rangierte Quellen und Informationen verlinkt wird. Als stellvertretender Indikator für „Popularität“ dient in dieser Untersuchung die Zahl der Backlinks, die ein Inhalt von anderen Inhalten erhält und der maßgeblich zu seinem Ranking in Suchmaschinen beiträgt.

Ein Popularitäts- oder eben PageRank-Effekt konnte im Greensill-Fall ebenfalls festgestellt werden (Wald $\chi^{2}=85.161, p<0.001$ ). Aus Tabelle 3 wird ersichtlich, dass 10000 zusätzliche Backlinks einen geringfügigen Einfluss auf die Verlinkungswahrscheinlichkeit einer Website mit Greensill-Inhalt haben $(\operatorname{Exp}(B)=1.001)$ - der Effekt wird erst bei größeren Mengen externer Verlinkungen spürbar. Mit anderen Worten: Neben den medienspezifischen Variablen des Hyperlinks und des Kanaltyps beeinflussen auch Backlinks (allerdings nur in größerer Anzahl) und mit ihnen Suchmaschinen über ihre Inhaltsauflistungen (Israel, Salomon 2018) das Audience Gatekeeping im Ereignisfall „Greensill“. 
Hypothese 1.3 geht von einer Wechselwirkung bzw. einem Interaktionseffekt (Kwon et al. 2012) zwischen Verlinkungshäufigkeit, Medientyp und Medienpopularität aus (vgl. Tabelle 3, Medium*Backlinks). Allerdings wirkt dieser PageRank-Effekt nicht generell: bei Fachmedien (Wald $\chi^{2}=0.819, p=0.366$ ) und etablierten Publikumsmedien (Wald $\chi^{2}=3.258, p=0.071$ ) ist der Interaktionseffekt nicht signifikant (vgl. Tabelle 3). Bei beiden Medientypen dürften also tiefer rangierte Suchergebnisse kompetenter Informationsanbieter gegenüber höherrangigen Suchergebnissen bevorzugt worden sein.

Anhand von Abbildung 2 lässt sich feststellen, dass PageRank-Effekte exponentiell wirken und dass die verschiedenen Medientypen unterschiedlich von ihnen profitieren, sich Domänen- und PageRank-Effekte also gegenseitig beeinflussen. Die steilsten Kurvengradienten weisen etablierte Wirtschaftstitel auf, bei ihnen erhöht Popularität die Verlinkungswahrscheinlichkeit von allen Medientypen am ausgeprägtesten (vgl. Abbildung 2).

Hypothese 1.2 und Hypothese 1.3 können ebenfalls bestätigt werden, letztere mit der Einschränkung, dass sich die Interaktionseffekte auf spezifische Medientypen beschränken und kompetente Publikums- und Fachmedien gegenüber höher rangierten Informationsquellen als Verlinkungen bevorzugt wurden.

In Beantwortung der Forschungsfrage 1 kann somit konstatiert werden, dass Audience Gatekeeping eine Aufmerksamkeitssteuerung zugunsten von Inhalten etablierter Medien, vor allem aus der Wirtschaftsdomäne fördert, indem die Selektivität der Links auch die Selektivität der Nutzung weiterer Quellen beeinflussen dürfte. Mit anderen Worten: Etablierte Medienstrukturen nehmen Einfluss auf die Informationsauswahl auch in den Sozialen Medien, zumindest im Ereignisfall Greensill. Nicht etablierte Medien, aber auch organisationale Stimmen (Parteien, Kommunen, Unternehmen) werden im vorliegenden Audience-Gatekeeping-Prozess wenig berücksichtigt.

\subsection{Rezyklierungs-Effekt: Zweitverwertung von Medieninhalten als dominantes Muster}

Forschungsfrage 2 fragt, ob Domänen- und Popularitätseffekte und ihre Interaktion auf selbstproduzierte Inhalte zurückgehen. Gerade Medien als aktive Akteure in AudienceGatekeeping-Prozessen verfügen über einen großen Vorrat an Informationen, aus denen sie sich bedienen können.

Hypothese 2.1 postuliert einen solchen Rezyklierungs- oder Eigenverlinkungs-Effekt, der sich durch Abgleich von Screen-Name und Hyperlink feststellen lässt. ${ }^{4}$ Von den 6449 untersuchten Tweets führen 1316 Verlinkungen $(20.4 \%$ ) auf eigenen Content. Es fällt auf, dass es zwischen Eigenverlinkung und Account-Typ (privat versus institutionell) signifikante Zusammenhänge $\left(\chi^{2}=971.671, p<0.001\right)$ mit nicht-trivialer Effektstärke $(\phi=0.387, p<0.001)$ bestehen, wobei bei der Bewertung der Effektstärke wiederum die hohe Fallzahl zu berücksichtigen ist.

4 Mittels String-Matching wurde festgestellt, ob der Hyperlink Personen- oder Firmennamen enthält, die entweder im user.screen_name oder in der user.description enthalten sind, oder vice versa. Personen- (PER) und Firmennamen (ORG) wurden mittels Spacy-Named Entity Recognition aus der Description extrahiert. Das Verfahren funktioniert zuverlässig, wenn auf eigene Personen- oder Firmenwebseiten oder auf den eigenen Twitter-Account verlinkt wird, da letzterer als Element den screen_name enthält. Bei Eigen-Verlinkungen auf andere Social-MediaKanäle (z. B. Youtube) ist eine linkbasierte Zuordnung jedoch nicht möglich, da der Identifikationsteil aus einem Token besteht. Eine Zufallsstichprobe von 2 Prozent der Fälle ergab eine Zuweisungsgenauigkeit (accuracy) von 91.5 Prozent. 
Abbildung 2: Kombinierte Darstellung der PageRank-und Interaktions-Effekte

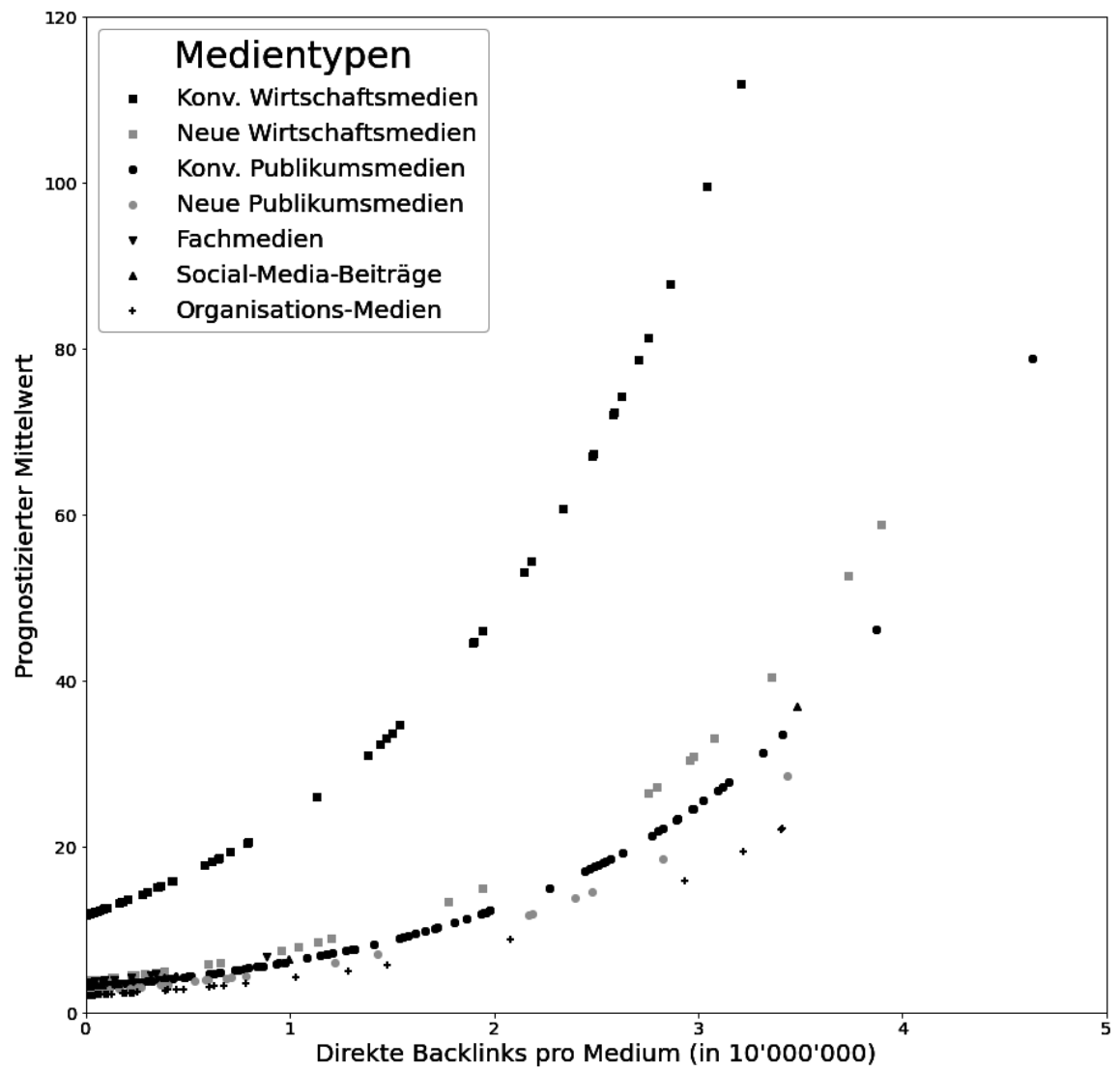

33.0 Prozent der Eigenverlinkungen stammen von privaten Accounts, 67.0 Prozent von institutionellen Accounts, die im Namen einer Organisation betrieben werden, wobei daran zu erinnern ist, dass private Accounts doppelt so viele Tweets abgesetzt haben wie institutionelle Accounts. Eigenverlinkung ist also eine Sache organisations-getriebener Kommunikation. Dies erscheint plausibel, da zwar theoretisch alle User die gleichen Chancen auf Eigenverlinkung haben (Ernste 2014), institutionelle Accounts aufgrund höherer Newsproduktion aber mehr Möglichkeiten. Besonders ausgeprägt ist die Eigenverlinkung bei den Medien: Von ihnen stammen nicht nur 73.0 Prozent aller institutionellen Tweets, sondern auch 85.9 Prozent aller institutionellen Eigenverlinkungen. Nicht mitgezählt bei diesem medialen Rezyklierungs-Effekt sind privat twitternde Medienschaffende, die auf das Medium ihres Arbeitgebers verweisen - sie sind bei den privaten Accounts erfasst. Die übrigen institutionellen Accounts, d. h. Firmen, Verbände, Kommunen und Parteien, steuern die verbleibenden 27.0 Prozent institutioneller Eigenverlinkungen bei, die allerdings nur zu 14.1 Prozent auf eigene, bereits veröffentlichte Inhalte führen.

Hypothese 2.1 kann somit bestätigt werden. 


\subsection{Potenz-Effekt: Extremverteilung der Aufmerksamkeit durch Audience Gatekeeping}

Hypothese 2.2 postuliert, dass Verlinkungen die zugänglichen Informationskanäle in einem Maße ungleich berücksichtigen, dass eine Extremverteilung resultiert, die an ihren Rändern - ähnlich einer extremen Pareto-Verteilung - sehr wenigen Medien sehr viel und in proportionalem Verhältnis sehr vielen Medien sehr wenig Aufmerksamkeit zuführt (Easley, Kleinberg 2010). Bei dieser als Power-Law oder Potenzgesetz bekannten Verteilung führt die Veränderung einer Größe zu einer proportionalen relativen Änderung einer anderen Größe - ähnlich der Flächenveränderung in einem Vieleck bei der Veränderung der Seitenverhältnisse.

Eine extreme Ungleichverteilung der Links wird bereits in der Frequenzanalyse sichtbar. 515 Quellen werden nur einmal verlinkt (54.6\% der Quellen mit zusammen $8.0 \%$ der Verweise) und 159 Quellen nur zweimal (16.9\% mit $4.9 \%$ Verweisen). Insgesamt erhalten 863 Quellen (91.6\%) weniger als 10 Verlinkungen. Am anderen Verteilungsrand erhalten die drei meistverlinkten Quellen - Financial Times, Bloomberg und Reuters - zusammen 1660 Verweise (0.3\% der Quellen mit $25.7 \%$ Verweisen), und die 10 am häufigsten verlinkten Quellen (1.1\%) zusammen 2689 Links $(41.7 \%)$.

Eine Endlastigkeits-Verteilung ist dann proportional (Milojevic 2010), wenn die Wahrscheinlichkeit, dass eine Webseite $k$-Links erhält, einem Potenzgesetz mit Exponent $r$ folgt (Barabási, Albert 1999). Statistisch lässt sich dies untersuchen, indem die Verteilstruktur auf eine negative lineare Beziehung zwischen den log-transformierten Werten von Verlinkungshäufigkeit und Anzahl Medien mit jeweils gleicher Verlinkungshäufigkeit getestet wird (Moody, White 2003, vgl. Abbildung 3).

Die Analyse der Verteilstruktur mittels Python-Modul powerlaw (Alstofft, Bullmore, Plenz 2014) zeigt eine signifikante Beziehung zwischen der Häufigkeit der Verlinkung $(k)$ und der Zahl der Medien mit der gleichen Verlinkungshäufigkeit (Medienfrequenz $M(k))$ mit $R^{2}=0.607, F(1,41)=63.407, p<0.001$, vgl. Abbildung 3). Das kollektive Ergebnis der Aufmerksamkeitslenkung durch Audience Gatekeeping weist auf eine Extremverteilung mit wenigen dominanten Quellen hin. Allerdings ist diese Ungleichverteilung weniger ausgeprägt als bei der Untersuchung von Kwon et al. (2012) und folgt nur moderat einer eindeutigen Power-Law-Verteilung (vgl. Abbildung 4, Empirische Daten). Ein Anpassungsgütetest (Alstofft et al. 2014) zeigt zudem, dass Power-Law die Verteilung der Verlinkungen zwar besser als eine Exponentialverteilung (LoglikelihoodRatio $R=22.1362, p=0.0102)$, aber geringfügig schlechter als eine trunkierte PowerLaw-Verteilung $(R=-0.4430, p=0.3466)$ oder eine Lognormalverteilung $(R=-0.2485$, $p=0.5878)$ beschreiben kann, allerdings ohne dass letztere sich als signifikant besseren Fit anbietet ( $p \geq 0.0500$, vgl. Abbildung 4).

Es kann also eine Extremverteilung angenommen werden, diese weist aber nicht alle erforderlichen Eigenschaften einer Power-Law-Verteilung auf, sondern entspricht tendenziell eher einer Lognormal-Verteilung mit rechtsschiefer Ausrichtung, deren Muster sich aus einer multiplikativen Überlagerung vieler kleiner Einflüsse („multiplikatives Rauschen") ergibt.

Hypothese 2.2 muss deshalb zurückgewiesen werden.

In Beantwortung von Forschungsfrage 2, die sich für den Grad der Aufmerksamkeitskonzentration auf wenige Titel interessiert, kann konstatiert werden, dass Audience Gatekeeping tatsächlich zu einer Extremverteilung von Aufmerksamkeit zugunsten weniger Titel führt. Welchen Anteil der Domänen-, PageRank-, Interaktions- und Rezyklierungs-Effekt im Einzelnen zu diesem Verteilmuster beigetragen haben, wäre in weiteren Untersuchungen zu klären. 
Abbildung 3: Negativer loglinearer Zusammenhang zwischen Verlinkungshäufigkeit und Medienfrequenz

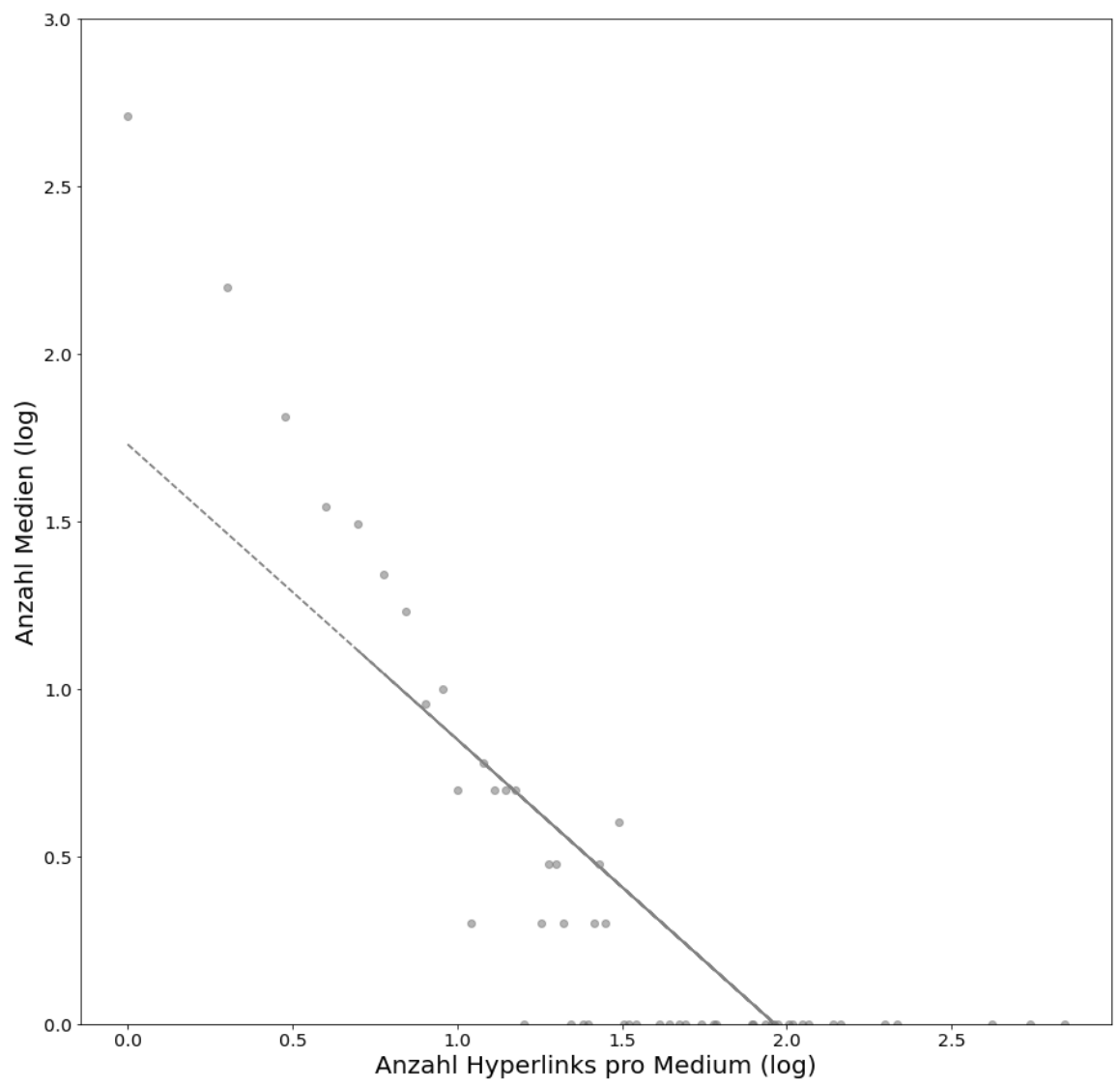

\section{Diskussion}

Wie verschiedene Autoren in theoretischen und praktischen Arbeiten zeigen konnten, erzeugen digitale Medienumgebungen einen Strukturwandel im Informationsauswahlund Agenda-Setting-Prozess. Ein Teil des Publikums übernimmt einen aktiven Part in der Aufmerksamkeitslenkung auf bestimmte Medieninhalte, und seine Selektionskriterien können erheblich von jenen professioneller Medienschaffender abweichen (Shoemaker, Johnson, Seon, Wang 2011). Audience Gatekeeping kann so eine alternative Themendarstellung etablieren, diese durchsetzen oder ein Thema überhaupt auf die Agenda bringen (Demirhan 2014; Meraz, Papacharissi 2013). Dies dürfte vor allem dann der Fall sein, wenn das besagte Thema von Beteiligten (mit-)inszeniert und berichtet und so unter dem Stichwort „Protest als Ereignis“ (Ertl 2015) subsumiert werden kann. Bei Ereignissen, die sich durch Nichtobtrusivität (Zucker 1978) auszeichnen, will heißen: zu dem sich Dritte nicht ohne Weiteres Informationen aus erster Hand beschaffen können, dürfte die Informationsabhängigkeit von medialer Recherche größer sein und die The- 
Abbildung 4: Anpassungsgütevergleich von Verteilfunktionen der Medienfrequenz

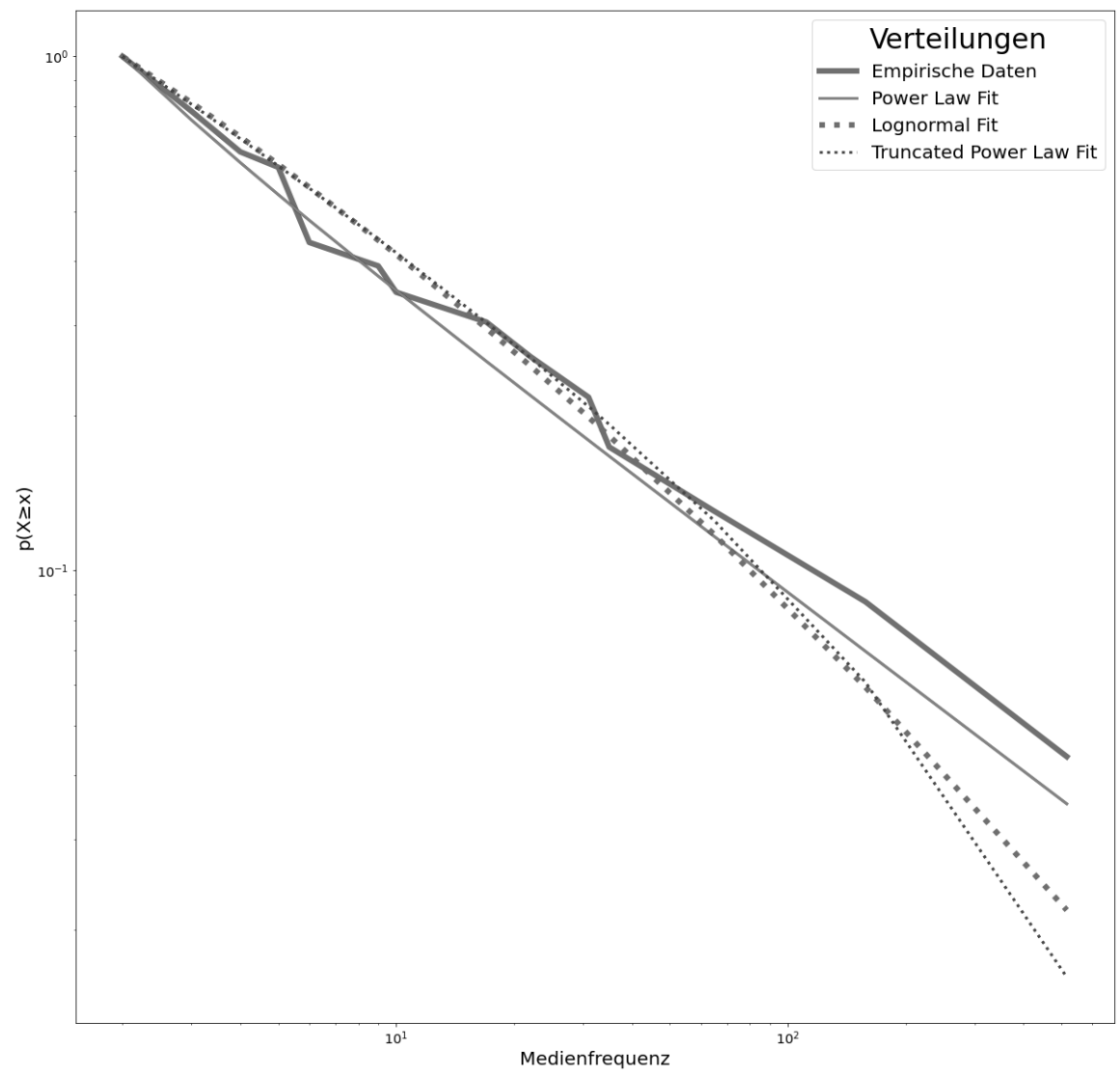

men(re)konstruktion weit weniger Varianz aufweisen. Untersucht wurden bisher ausschließlich politische Kommunikationsereignisse. Die Berichterstattung zu einem vielbeachteten Wirtschaftsereignis, der Insolvenz des Lieferkettenfinanzierers Greensill, liefert Hinweise darauf, dass ähnliche Prozesse auch in anderen Gesellschaftsbereichen wirksam sind.

Die Ergebnisse der Vergleichsstudie von Kwon et al. (2012) konnten weitgehend bestätigt werden: Das Gatekeeping des Twitter-Publikums wird durch Hyperlinkstrukturen und Medientypen (oder Domänen) beeinflusst. Allerdings waren die prominentesten Kanäle nicht Accounts aus den Sozialen Medien, sondern die etablierten Wirtschaftstitel, die bei der Darstellung und Interpretation der zum aktuellen Zeitpunkt undurchsichtigen Vorgänge offensichtlich ihre Professionalität in der Nachrichtenbeschaffung und ihre investigativen Vorteile ausspielen konnten. Ebenfalls zu relativieren ist der Befund der erstgenannten Studie, dass Soziale Medien im Ereignisfall Räume schaffen, in denen sich journalistisch aufbereitete Informationen etablierter Nachrichtenkanäle und nutzergenerierte Inhalte mischen - zumindest was Verlinkungen betrifft, werden 
hauptsächlich journalistische Informationen geteilt, auf nutzergenerierten Content wird weiter weniger häufig verlinkt. Das Fazit von Kwon et al. (2012), dass der GatekeepingProzess auf Twitter die Autorität etablierter Medien als primäre Informationslieferanten schmälern konnte, trifft zumindest auf diesen Ereignisfall nicht zu: Gemessen an den Verlinkungen haben etablierte Nachrichtenkanäle ihren Rang als vorherrschende Agenda-Setter behauptet. Soziale Berichterstattung durch neue Plattformen führt demnach weder zwangsläufig zu einer Fragmentierung der Medien noch zu einem Umsturz in der Gatekeeping-Hierarchie. Sie kann auch Medienkonzentration und bestehende Gatekeeping-Muster verstärken.

Hingegen kommt die vorliegende Untersuchung unisono mit Kwon et al. zum Schluss, dass Suchmaschinen einen nicht unerheblichen Einfluss auf die Informationsauswahl haben, da Backlinks als weitere Variable das Audience Gatekeeping beeinflussen. Die beschriebenen Konzentrationseffekte, ausgelöst durch Präferenzen für bestimmte (wirtschaftsorientierte) Medientypen, durch Suchmaschinenergebnisse und die Interaktion zwischen diesen Effekten, erzeugt auf kollektiver Ebene Selektionsmuster mit einem hohen Ungleichgewicht: Audience Gatekeeping schafft Aufmerksamkeitsstrukturen mit sehr wenigen sehr hochsichtbaren und sehr vielen sehr wenig sichtbaren Informationslieferanten. Damit widerspiegelt sich in beiden Untersuchungen das LongTail-Paradox des Internets, das ein zugleich stark konzentriertes und ebenso stark diversifiziertes Informationsangebot erzeugt. Dass dieses Paradox die mathematische Form einer Power-Law-Verteilung der digitalen Information annimmt, konnte mit der vorliegenden Untersuchung jedoch nicht bestätigt werden.

Eine Untersuchung besonders eines nicht generalisierbaren Einzelfalls erzeugt gewöhnlich mehr Fragen, als sie Antworten liefern kann. Immerhin ergeben sich daraus Anstöße für weitere Forschungsvorhaben, und es seien im Folgenden vier mögliche Schwerpunkte weiterer Audience-Gatekeeping-Forschung genannt, speziell natürlich im Bereich von Wirtschaftskommunikation:

\subsection{Ereignistypologie als Ansatz für eine dynamische Gatekeeping-Theorie}

Mit der Untersuchung eines einzelnen Wirtschaftsereignisses lassen sich weder die These einer alternativen Ereignisdarstellung durch Audience Gatekeeping noch die These einer bloßen Resonanzverstärkung etablierter Informationsstrukturen durch Publikumsaktivitäten verallgemeinern. Auch eine These, dass Ereignisse Aufmerksamkeit bündeln und deshalb als Plattformen zur Bekanntheitssteigerung genutzt werden, sollte anhand unterschiedlicher Ereignisse verifiziert werden. Dazu braucht es eine Typologie von Ereignissen, anhand derer Muster und Effekte des Audience Gatekeeping in verschiedenen Gesellschaftsbereichen - Politik, Wirtschaft, Sport, Gesundheitswesen - erhoben werden können.

Gut möglich, dass alternative Themen(durch)setzungen bei „obtrusiven“ Wirtschaftsereignissen, wie Arbeitsstreiks, Produktboykotten oder Standortschließungen mit Angestellten- und Kundenbeteiligung, eher anzutreffen sind. Ebenso möglich, dass bei nicht-obtrusiven Ereignissen der Kabinettspolitik das Audience Gatekeeping mit der Berichterstattung der etablierten Medien (oder der politischen Public-Relations-Abteilungen) vorlieb nehmen muss und deren Themenstrukturierung übernimmt. Eine Typologie könnte in diesem Zusammenhang festhalten, ob im Ereignisfall Audience Gatekeeping zu alternativen Thematisierungen und in der Folge zu einem breiter gefächerten Agenda Setting führt. 


\subsection{Differenzierte Untersuchungen zur Erklärung der Ereigniswahrnehmung}

Wie auch in anderen Untersuchungen, so fällt in den Greensill-Daten die Diskrepanz auf zwischen der hohen Zahl der Medien, auf die verlinkt wird (Long Tail) und der geringen Zahl von Medien, die den Großteil der Links auf sich ziehen. Ein hypothetisch breites Informationsangebot fördert faktisch kaum Informationsalternativen, es sind wenige Schlüsselmedien, die eine spezifische Ereigniswahrnehmung erzeugen, was nicht ohne Rückkoppelungseffekte auf die Ereignislage bleiben dürfte.

Allerdings zeigen die erhobenen Domänen- und PageRank-Effekte sowie der Interaktions-Effekt als Verbindung zwischen diesen beiden Effekten, dass diese Diskrepanz durch Routinen und Präferenzen des Publikums mitverursacht wird. Künftige Untersuchungen könnten sich noch stärker mit der Vielzahl jener als „multiplikatives Rauschen“ bezeichneten kleinen Effekte befassen, die als Zusammenspiel bestimmter Informationsstrukturen und -routinen bei Urhebern, Berichterstattern und Empfängern zu extremen Konzentrationsprozessen in der Informationsverarbeitung führen. Solche Untersuchungen müssten inhaltlich und in der Untersuchung von Netzwerkstrukturen und -prozessen erheblich weitergehen als die vorliegende, die sich damit begnügt hat, eine singuläre Informationseinheit, den Hyperlink, auf Verteilmuster hin zu untersuchen. Verlinkungen sind allerdings nur in etwa der Hälfte der zirkulierten Posts zu Greensill enthalten und machen auch dort nur einen Bruchteil des Informationsaustauschs aus. Weitere Studien müssten deshalb auch die eigentlichen Inhalte der Ereigniskommunikation erfassen.

\subsection{Berücksichtigung von Plattformisierungs-Effekten}

Beachtung verdient auch das Phänomen der indirekten Verlinkung, d. h. die Verlinkung auf Informationen auf den Account einer - in den Worten des fög (2019): ungebündelten - Social-Media-Plattform, die ihrerseits per Link an eine Information eines gebündelten Informationsanbieters weiterleitet. Es stellt sich nicht nur die Frage des Markeneffekts, sondern auch der Qualitätswahrnehmung und Glaubwürdigkeit solcher indirekten Audience Gatekeepings: Bereits direktes Audience Gatekeeping hat den Effekt, dass „die Nutzer und Nutzerinnen [...] sich bestenfalls [erinnern], dass sie einen bestimmten Beitrag z. B. auf einer bestimmten Plattform (z. B. Facebook) gesehen haben, nicht aber, dass er z. B. von tagesanzeiger.ch oder Blick.ch stammt“ (fög 2019: 17). Wenn bereits solche Vorgänge die Bindung an die etablierten Medienmarken (Kalogeropoulos, Newman 2017), das Qualitätsbewusstsein und die Bereitschaft schwächen, für qualitativ hochwertige Informationen Zeit und Geld zu investieren (Reuters Institute 2019), dann dürften diese Effekte beim indirekten Gatekeeping, das in dieser Untersuchung ein Sechstel des Verlinkungsvolumens umfasste, noch stärker ausfallen.

Aus forschungsökonomischen und methodischen Gründen konnte dieser besonderen Form des Audience Gatekeeping kein Platz eingeräumt werden. Um die Selektionswirkungen des Audience Gatekeeping genau zu erfassen, ist es jedoch unerlässlich, die Intentionen des Audience Gatekeepers zu kennen - beispielsweise seine Trade-offÜberlegungen zwischen Verbreitungseffizienz und Inhaltswirkung (lohnt sich der Aufwand, direkt zum intendierten Originalbeitrag zu verlinken? Oder, auch möglich: spielt dieser überhaupt eine Rolle?). Eine solche Untersuchung könnte zweifellos zur erwähnten differenzierten Erklärung der Ereigniswahrnehmung beitragen. 


\subsection{Erbebung von Rekonfigurations-Effekten}

Eine punktuelle Untersuchung wie die vorliegende kann wenig dazu beitragen, die Effekte der Selektions- „Rekonfiguration“ im Mediensystem zu bewerten. Der Ereignisfall stützt eher die Position von Neuman, Bimber, Hindman (2011), Karpf (2010) und Hindman (2008), dass sich alte Informationshierarchien in digitalen (Wirtschafts-)Medien im neuen Gewand zeigen. Hier wäre eine Kontrastierung mit Gegenbeispielen interessant, in denen „networked publics“ (Meraz, Papacharissi 2013) die Spielregeln des Informationsmarktes verändern konnten. Die Fälle von Gamestop und AMC deuten an, dass vernetzte Publika in Form von Reddit-Raiders erheblichen Einfluss auf das Börsengeschehen nehmen können.

Auch mangelt es in der Wirtschaft noch an Studien zum Ausmaß an Falschinformationen, die von vernetzten Publika oder Einzelakteuren eingeschleust werden (Marantz, 2019; Pomerantsev, 2019; Singer, Brooking, 2018) - nicht nur Politik und Gesundheitswesen sind von diesem Phänomen betroffen. Studien zu Aktienmarkt-Manipulationen (z. B. Siering, Muntermann, Grčar 2021) können den Weg zu Einblicken in Strukturen und Prozesse manipulativer Arten von wirtschaftlichem Gatekeeping ebnen.

\section{Literatur}

Alstofft, J., Bullmore, E., Plenz, D. (2014). powerlaw: A Python Package for Analysis of Heavytailed Distributions. PloS ONE 9(1): e85777. https://doi.org/10.1371/journal.pone.0085777.

Backhaus, K., Erichson, B., Plinke, W., Weiber, R. (2016). Multivariate Analysemethoden. Berlin/ Heidelberg: Springer Verlag.

Banerjee, A., Choudhary, A. (2017). Advanced Page Rank Algorithm with Semantics, In Links, Out Links and Google Analytics. International Journal of Computer Trends and Technology, 50(3), S. 137-142.

Barabási, A.-L., Albert, R. (1999). Emergence of Scaling in Random Networks. Science, 286(5439), S. 509-512.

Barzilai-Nahon, K. (2008). Toward a Theory of Network Gatekeeping: A Framework for Exploring Information Control. Journal of the American Society for Information Science and Technology, 59(9), S. 1493-1512.

Barzilai-Nahon, K. (2009). Gatekeeping: A Critical Review. Annual Review of Information Science and Technology, 43(1), S. 1-79.

Benkler, Y. (2006). The Wealth of Networks: How Social Production Transforms Markets and Freedom. Yale University Press.

Bharathi S., Geetha A. (2017). Sentiment Analysis for Effective Stock Market Prediction. International Journal of Intelligent Engineering and Systems, 10(3), S. 146-153.

Bharathi, S., Geetha, A., Sathiynarayanan, R. (2017). Sentiment Analysis of Twitter and RSS News Feeds and Its Impact on Stock Market Prediction. International Journal of Intelligent Engineering and Systems, 10(6), S. 68-77.

Birkland, T. (1997). After Disaster. Agenda Setting, Public Policy, and Focusing Events. Washington: Georgetown Press.

Blume, F., Kröner, A., Volkery, C. (2021). Greensill-Rettung ist gescheitert - Aufstieg und Fall eines Fintech-Stars. Handelsblatt, Ausgabe vom 14.03.2021, https: //www.handelsblatt.com/ finanzen/banken-versicherungen/banken/finanzskandal-greensill-rettung-ist-gescheitert-auf stieg-und-fall-eines-fintech-stars [02.11.2021].

Bytzek, E. (2008). Ereignisse und ihre Wirkung auf die Themenagenda der Wählerschaft. Medien E Kommunikationswissenschaft, 56(3-4), S. 433-451.

Caliñgo, A., Sison, A., Tanguilig, B. (2016). Prediction Model of the Stock Market Index Using Twitter Sentiment Analysis. International Journal of Information Technology and Computer Science, 8(10), S. 11-21. 
Case 1: 18-cv-01669-UNA (2018). Weintraub vs. Tesla. https: //images.law.com/contrib/content/ uploads/documents/394/3448/Musk-Derivative-Complaint.pdf [02.11.2021].

Cha, M., Haddadi, H., Benevenuto, F., Gummadi, K. (2010). Measuring User Influence in Twitter: The Million Follower Fallacy. Proceedings of the Fourth International AAAI Conference on Weblogs and Social Media, S. 10-17.

Chakraborty, A., Ghosh, S., Ganguly, N., Gummadi, K. (2019). Editorial Versus Audience Gatekeeping: Analyzing News Selection and Consumption Dynamics in Online News Media. IEEE Transactions on Computational Social Systems, 6(4), S. 680-691.

Coddington, M., Holton, A. (2014). When the Gates Swing Open: Examining Network Gatekeeping in a Social Media Setting. Mass Communication and Society, 17(2), S. 236-257. https:// doi.org/10.1080/15205436.2013.779717.

Coxe, S., West, S., Aiken, L. (2009). The Analysis of Count Data: A Gentle Introduction to Poisson Regression and its Alternatives. Journal of Personality Assessment, 91, S. 121-136.

Da, Z., Engelberg, J., Gao, P. (2011). In Search of Attention. The Journal of Finance, 66(5), S. 14611499.

Demirhan, K. (2014). Social Media Effects on the Gezi Park Movement in Turkey: Politics under Hashtags. In: B. Patrut, M. Patrut (Hrsg.), Social Media in Politics. Springer International Publishing, S. 281-314.

Dimitrova, D., Connolly-Ahern, C., Williams, A., Kaid, L., Reid, A. (2003). Hyperlinking as Gatekeeping: Online Newspaper Coverage on an Execution of the American Terrorist. Journalism Studies 4(3), S. 401-414.

Easley, D., Kleinberg, J. (2010). Networks, Crowds, and Markets: Reasoning about a Highly Connected World. Cambridge University Press.

Ernste, T. (2014). The Networked Gatekeeping Process for News in the 21st Century. International Conference on Collaboration Technologies and Systems (CTS) proceedings, S. 11-18.

Ertl, S. (2015). Protest als Ereignis - Zur medialen Inszenierung von Bürgerpartizipation. transcript Verlag.

Ferber, M. (2021). Das billige Geld der Zentralbanken zieht Spieler an. Neue Zürcher Zeitung, 30.03., S. 19.

Fletcher R., Park S. (2017). The Impact of Trust in the News Media on Online News Consumption and Participation. DigitalJournalism, S. 1-19.

fög - Forschungsinstitut Öffentlichkeit und Gesellschaft (2019). Qualität der Medien. Schwabe Verlag.

Förster, M., Happ, R., Walstad, W. (2019). Relations between Young Adults' Knowledge and Understanding, Experiences, and Information Behaviour in Personal Finance Matters. Empirical Research in Vocational Education and Training, 11(2), S. 1-21.

Freelon, D., \& Wells, C. (2020). Disinformation as Political Communication. Political Communication, 37(2), S. 145-156.

Goode, L. (2009). Social News, Citizen Journalism, and Democracy. New Media E Society, 11(8), S. 1287-1305.

Grant \& Eisenhofer (2019). Grant \& Eisenhofer Files Suit Seeking to Block Elon Musk from Tweeting About Tesla. https: //www.gelaw.com/ge/press-release/Tesla_03-08-19.pdf [02.11.2021].

Halavais, A. (2008). The Hyperlink as Organizing Principle. In: J. Turrow and L. Tsui (Hrsg.), The Hyperlinked Society. Ann Arbor: The University of Michigan Press, S. 56-69.

Hilbe, J. (2014). Negative Binomial Regression. Cambridge University Press.

Hindman, M. (2008). The Myth of Digital Democracy. Princeton University Press.

Honegger, L. (2021). Was steckt hinter Elon Musks Bitcoin-Kehrtwende? NZZ, 15.05., S. 25.

Hoskins, A., O’Loughlin, B. (2011). Remediating Jihad for Western News Audiences: The Renewal of Gatekeeping? Journalism 12(2), S. 199-216.

Hughes, H. (1980). News and the Human Interest Story. New York: Routledge.

Israel, O., Samson, O. (2018). The Impact of Search Engines in the World Today. International Journal of Management, IT E Engineering, 8(3), 10-22.

Jain, A., Dave, M. (2013). The Role of Backlinks in Search Engine Ranking. International Journal of Advanced Research in Computer Science and Software Engineering, 3(4), S. 596-599. 
Jungherr, A., Schroeder, R. (2021). Disinformation and the Structural Transformations of the Public Arena. Addressing the Actual Challenges to Democracy. Social Media + Society, JanuaryMarch, S. 1-13.

Jürgens, P., Jungherr, A., Schoen, H. (2011). Small Worlds with a Difference: New Gatekeepers and the Filtering of Political Information on Twitter. In: Proceedings of the 3rd International Web Science Conference, 21: 1-21: 5. WebSci '11. New York, NY, USA: ACM. https://doi.org/ $10.1145 / 2527031.2527034$.

Kalogeropoulus, A., Newman, N. (2017). „I Saw the News on Facebook“. Brand Attribution when Accessing News from Distributed Environments. reutersinstitute.politics.ox.ac.uk/sites/de fault/files/2017-07/Brand\%20attributions\%20report.pdf [02.11.2021].

Karpf, D. (2010). Macaca Moments Reconsidered: Electoral Panopticon or Netroots Mobilization? Journal of Information Technology E Politics, 7(2-3), S. 143-162.

Kepplinger, H. (2001). Der Ereignisbegriff in der Publizistikwissenschaft. Publizistik, 46, S. 117139.

Keyling, T. (2017). Kollektives Gatekeeping. Springer Fachmedien: Wiesbaden.

Kwon, K., Oh, O., Agrawal, M., Rao, H. (2012). Audience Gatekeeping in the Twitter Service: An Investigation of Tweets about the 2009 Gaza Conflict. AIS Transactions on Human-Computer Interaction, 4(4). S. 212-229.

Leavitt, A., Robinson, J. (2017). The Role of Information Visibility in Network Gatekeeping: Information Aggregation on Reddit during Crisis Events. Proceedings of the ACM Conference on Computer Supported Cooperative Work, S. 1246-1261.

Lindmayer, K., Dietz, H. (2001). Informationsquellen für Anleger. In: Geldanlage und Steuer 2002. Gabler Verlag, Wiesbaden, S. 523-525.

Marantz, A. (2019). Antisocial: Online Extremists, Techno-Utopians, and the Hijacking of the American Conversation. Viking.

Martin, J. (2001): Für eine Typologie der „Ereignisse“: Das Beispiel des Vendéekrieges. In: A. Suter, M. Hettling (Hrsg.), Struktur und Ereignis (Sonderheft 19, Geschichte und Gesellschaft. Zeitschrift für Historische Sozialwissenschaft). Göttingen: Vandenhoeck \& Ruprecht, S. 208-223.

McCombs, M. (2004). Setting the Agenda. The Mass Media and Public Opinion. Cambridge: Polity Press.

Mehrotra, K. (2017). The Crossroads and Convergences of Media and Technology Companies. Facebook as the Latest Media Gatekeeper. The SOAS Journal of Postgraduate Research, vol. 10, S. 9-24.

Meraz, S., Papacharissi, Z. (2013). Networked Gatekeeping and Networked Framing on \#Egypt. The International Journal of Press/Politics, 18(2), S. 138-166.

Meraz, S., Papacharissi, Z. (2016). Networked Framing and Gatekeeping. In: T. Witschge, C. Anderson, D. Domingo, A. Hermida (Hrsg.), The Sage Handbook of Digital Journalism. SAGE, S. $95-112$.

Milojevic, S. (2010). Power-law Distributions in Information Science - Making the Case for Logarithmic Binning. Journal of the American Society for Information Science and Technology, 61(12), S. 2417-2425.

Mitchelstein, E., Boczkowski, P. (2009). Between Tradition and Change - A Review of Recent Research on Online News Production. Journalism, 10(5), S. 562-86.

Moody, J., White, D. (2003). Structural Cohesion and Embeddedness: A Hierarchical Concept of Social Groups. American Sociological Review, 68(1), S. 103-127.

Mulvaney, T., Baldo, M., Swerdlow, M., Cavaliere, F. (2018). Social Media and the C-Suite: The Ethical and Legal Implications. Southwestern Business Administration Journal, 17(1), Article 2.

Nelson, M., Wooditch, A., Dario, L. (2014): Sample Size, Effect Size, and Statistical Power: A Replication Study of Weisburd's Paradox. Journal of Experimental Criminology, preliminary draft, S. 1-41.

Neuman, W., Bimber, B., Hindman, M. (2011). The Internet and Four Dimensions of Citizenship. In: R. Shapiro, L. Jacobs (Hrsg.), The Oxford Handbook of American Public Opinion and the Media. Oxford University Press, S. 22-42.

Neuman, R., Guggenheim, L., Jang, S., Bae, S. (2014). The dynamics of public attention: Agendasetting theory meets big data. Journal of Communication, 64(2), S. 193-214. 
Nielsen, R. (2014). Varieties of Online Gatekeeping. Rethinking Journalism II - proceedings, S. 129.

Osborne, J. (2017). Regression and Linear Modeling: Best Practices and Modern Methods. Los Angeles, CA: Sage.

Pałka-Suchojad, K. (2021). Who Keeps the Gate? Digital Gatekeeping in New Media. Zeszyty PRASOZNAWCZE, 64(2), S. 91-99.

Patel, S., Trivedi, D., Bhatt, A., Shanti, C. (2021). Web Visibility and Research Productivity of NIRF Ranked Universities in India: A Webometric Study. Library Philosophy and Practice (e-journal), 5326, o. S.

Payne, E., Gebregziabher, M., Hardin, J., Ramakrishnan, V., Egede, L. (2017). An Empirical Approach to Determine a Threshold for Identification of Overdispersion in Count Data. Communications in Statistics - Simulation and Computation, 47, S. 1722-1738.

Pollock, T., Rindova, V. (2003). Media Legitimation Effects in the Market for Initial Public Offerings. The Academy of Management Journal, 46, S. 631-642.

Pomerantsev, P. (2019). This is not Propaganda: Adventures in the War against Reality. Faber \& Faber.

Poonkuzhali G., Kishore Kumar R., Sudhakar P., Uma G., Sarukesi K. (2012). Relevance Ranking and Evaluation of Search Results through Web Content Mining. IMECS-Proceedings, Vol. 1, o.P.

Ranjan, S., Sood, S. (2019). Social Network Investor Sentiments for Predicting Stock Price Trends. International Journal of Scientific Research and Review, 07(02), S. 90-97.

Reddy, P. (1991). Aufmerksamkeit und das Erlernen von Fertigkeiten. In: J. Gerstenmaier (Hrsg.), Einführung in die Kognitionspsychologie. München u. a.: UTB Wissenschaft, S. 91-119.

Reuters Institute (2019). Digital News Report 2019. Oxford: University of Oxford; reutersinsti tute.politics.ox.ac.uk/sites/default/files/2019-06/DNR_2019_FINAL_0.pdf [02.11.2021].

Riffe, D., Reader, B. (2007). Most Rely on Newspapers for Local Business News. Newspaper Research Journal, S. 82-98

Schneider, J., Eisenegger, M. (2020). Mediennutzung und persönliche Themenagenda - wie das Newsrepertoire die Wahrnehmung von Kommunikationsereignissen prägt. In: fög (Hrsg.), Qualität der Medien Jabrbuch 2020. Basel: Schwabe Verlag, S. 101-114.

Schöchli, H. (2021). Braucht es wegen der CS schärfere Bankregeln? NZZ, S. Juni, S. 24.

Schwaiger, L., Schneider, J., Vogler, D. (2020). Mediennutzung. In: fög (Hrsg.), Qualität der Medien Jabrbuch 2020. Basel: Schwabe Verlag, S. 135-139.

Setiawan, A., Harahap, Z., Syamsuar, D., Kunang, Y.(2020). The Optimization of Website Visibility and Traffic by Implementing Search Engine Optimization (SEO). CommIT Journal, 14(1), S. 31-44.

Shoemaker, P., Johnson, P., Seon, H., Wang, X. (2011). Readers as Gatekeepers of Online News: Brazil, China, and the United States. Brazilian Journalism Research, 6(1), S. 55-77.

Siering, M., Muntermann, J., Grčar, M. (2021). Design Principles for Robust Fraud Detection: The Case of Stock Market Manipulations. Journal of the Association for Information Systems, 22(1), S. 156-170.

Singer, P., Brooking, E. (2018). Like War: The Weaponization of Social Media. Eamon Dolan.

Smyrnaios, N. (2012). How Does News Infomediation Operate Online? The Examples of Google and Facebook. WMEMC-proceedings, S. 1-17.

Soroka, S. (2002). Agenda Setting Dynamics in Canada. Vancouver: UBC Press.

Stiglitz, J. (2002).Transparency in Government. In: World Bank Institute (Eds.), The Right to Tell: The Role of Mass Media in Economic Development. Washington, S. 27-44.

Strauss, N., Vliegenthart, R., Verhoeven, P. (2017). Intraday News Trading: The Reciprocal Relationships Between the Stock Market and Economic News. Communication Research, 45(7), S. 1054-1077.

Strauss, S. (2018). Network Gatekeeping on Twitter during the German National Election Campaign 2017. In: 14. Düsseldorfer Forum Politische Kommunikation, Extended Abstract, S. 1-7.

Strycharz, J., Strauss, N., Trilling, D. (2018). The Role of Media Coverage in Explaining Stock Market Fluctuations: Insights for Strategic Financial Communication. International Journal of Strategic Communication, 12(1), S. 67-85. 
Suksida, T., Santiworarak, L. (2017). A Study of Website Visibility in Webometrics Ranking of World University by Using SEO Tool. ICLEHI-proceedings, S. 135-142.

Sun, T., Wang, J., Zhang, P., Cao, Y., Liu, B., Wang, D. (2017). Predicting Stock Price Returns Using Microblog Sentiment for Chinese Stock Market. In: 3rd International Conference on Big Data Computing and Communications, BigCom Proceedings, S. 87-96.

Tetlock, P. (2007). Giving Content to Investor Sentiment: The Role of Media in the Stock Market. The Journal of Finance, 62, S. 1139-1169.

Tetlock, P. (2014). Information Transmission in Finance. Annual Review of Financial Economics, 6(1), S. 365-384.

Vos, T. (2015). Revisiting Gatekeeping Theory during a Time of Transition. In: T. Vos, F. Heinderyckx (Eds.), Gatekeeping in Transition. Routledge, S. 17-38.

Wahyuningrum, T., Pandiya, R. (2017). Logaritmic Fuzzy Preference Programming Approach for Evaluating University Ranking Optimization. Scientific Journal of Informatics, 4(1), S. 1-7.

Welbers, K. (2018). Social Media Gatekeeping: An Analysis of the Gatekeeping Influence of Newspapers' Public Facebook Pages. New Media E Society, 20(12), S. 4728-4747.

Wiedel, F. (2015). Audience is King. Brand is King Kong. Eine qualitative Interview-Reihe mit leitenden Nachrichtenjournalisten zu den Akteur-Struktur-Dynamiken und Arbeitslogiken im deutschen Mediensystem. Blogbeitrag, https://medialogic.hypotheses.org/167 [02.11.2021].

Wilson, S. (2021). What's Behind the Collapse of Greensill Capital, and Why Does it Matter? Moneyweek, 13.03., moneyweek.com/investments/stockmarkets/uk-stockmarkets/602920/ whats-behind-the-collapse-of-greensill-capital-and [02.11.2021].

$\mathrm{Xu}, \mathrm{W} .$, Feng, M. (2014). Talking to the Broadcasters on Twitter: Networked Gatekeeping in Twitter. Conversations with Journalists. Journal of Broadcasting E Electronic Media, 58(3), S. 420437.

Zhao, B., He, Y., Yuan, C., Huang, Y. (2016). Stock Market Prediction Exploiting Microblog Sentiment Analysis. Proceedings of 2016 International Joint Conference on Neural Networks (IJCNN) IEEE, S. 4482-4488.

Ziakis, C., Vlachopoulou, M., Kyrkoudis, T., Karagkiozidou, M. (2019). Important Factors for Improving Google Search Rank. Future Internet, 11, 32, S. 1-2.

Zucker, H. (1978). The Variable Nature of News Media Influence. In: B. Ruben (Hrsg.), Communication Yearbook, Vol. 2. New Brunswick: Transaction Books, S. 225-240.

(C) Stefan Gürtler 